\title{
Provoking factors for postpartum chronic hypertension in women with preceding gestational hypertension/preeclampsia: A longitudinal cohort study of 22,798 pregnancies
}

\author{
Kuo-Hu Chen ${ }^{1,2, \bowtie}$, and Li-Ru Chen ${ }^{3,4}$ \\ 1. Department of Obstetrics and Gynecology, Taipei Tzu-Chi Hospital, The Buddhist Tzu-Chi Medical Foundation, Taipei, Taiwan \\ 2. School of Medicine, Tzu-Chi University, Hualien, Taiwan \\ 3. Department of Physical Medicine and Rehabilitation, Mackay Memorial Hospital, Taipei, Taiwan \\ 4. Department of Mechanical Engineering, National Chiao-Tung University, Hsinchu, Taiwan
}

$\triangle$ Corresponding author: Professor Kuo-Hu Chen, Department of Obstetrics and Gynecology, Taipei Tzu-Chi Hospital, The Buddhist Tzu-Chi Medical Foundation, No. 289, Jianguo Road, Xindian, New Taipei City, Taiwan. Telephone: 886-2-66289779; Fax: 886-2-66289009; E-mail address: alexgfctw@yahoo.com.tw

() The author(s). This is an open access article distributed under the terms of the Creative Commons Attribution License (https://creativecommons.org/licenses/by/4.0/). See http://ivyspring.com/terms for full terms and conditions.

Received: 2019.08.18; Accepted: 2020.01.24; Published: 2020.02.10

\begin{abstract}
Background: A proportion of women with pregnancies complicated by gestational hypertension/preeclampsia (GH-PE) will have persistent postpartum chronic hypertension (CHTN). Common risk factors for postpartum CHTN include older age, pre-existing $\mathrm{CHTN}$, smoking, pre-pregnancy obesity (elevated BMI), and co-morbidities such as thyroid disorders. However, most of explored risk factors are pre-pregnancy factors, and were mainly based on studies with small sample size.

Methods: To investigate provoking pre-pregnancy and intra-pregnancy factors for postpartum CHTN in women with preceding GH-PE, the cohort study enrolled 22,798 index pregnancies to analyze individual characteristics, co-morbidities and postpartum outcomes after excluding women with pre-existing $\mathrm{CHTN}$.

Results: Among 2,132 GH-PE pregnancies, 428 (20.1\%) were complicated with postpartum CHTN. After adjustment, logistic regression analysis revealed excessive pregnant weight gain $(\geq 10 \mathrm{kgw}$ at 28 weeks' gestation) (OR: 14.50, 95\% Cl: 11.02-19.08) and gestational diabetes mellitus (GDM) (OR: 6.25, 95\% Cl: 4.98-7.85) were major risk factors for developing CHTN, other than age (OR: 1.80, 95\% Cl: 1.68-1.93), pre-pregnancy BMI (OR: $3.15,95 \% \mathrm{Cl}$ : 2.75-3.60), severity of GH-PE (OR: 2.46, $95 \% \mathrm{Cl}$ : 1.97-3.07), smoking (OR: $1.79,95 \% \mathrm{Cl}: 1.35-2.38$ ), and overt DM (OR: $2.30,95 \% \mathrm{Cl}$ : 1.73-3.06).

Conclusion: Excessive pregnant weight gain and GDM are major intra-pregnancy risk factors for postpartum CHTN in women with preceding GH-PE. Future studies should investigate interventions such as a healthy diet, appropriate physical exercise and avoidance of excessive pregnant weight gain as a means to reduce the frequency of $\mathrm{CHTN}$ following pregnancy.
\end{abstract}

Key words: gestational hypertension; preeclampsia; chronic hypertension; pregnant weight gain; gestational diabetes mellitus

\section{Introduction}

In the United States, gestational hypertension/ preeclampsia (GH-PE) is the most common medical complication during pregnancy, with an overall prevalence of $6 \%-8 \%$ [1]. PE is one of the main causes 
of maternal and perinatal morbidity and mortality, especially in low- and middle-income countries [2-4], and GH-PE predisposes mothers and fetuses to cardiovascular disease later in life [3, 5-8]. The etiology of PE is unclear. However, a two-stage model of abnormal trophoblastic invasion during placental implantation, leading to placenta ischemia and insufficiency, with subsequent endothelial injury, is believed to underlie the pathophysiology of GH-PE $[1,2,9-11]$. In consequence, endothelial cells may be injured by a combination of factors induced by placental hypoxia and an abnormal maternal inflammatory response [12]. Endothelial dysfunction leads to abnormal vasoconstriction, increased vascular permeability, and thrombosis, which induce systemic hypertension, edema, and systemic and placental infarcts, respectively [12]. Elevated BP may be related to decreased production of nitrogen oxide (NO) [2], leading to endothelial dysfunction and decreased vasodilatatory responses. Chronic inflammation, oxidative stress, insulin resistance and endothelial cell activation related to pregnancy have been described in obese, diabetic or pregnant women, and have been associated with hypertension in both pregnant and non-pregnant women [10, 12, 13-17]. During pregnancy, abnormal morphological and blood-flow changes of placenta, including preterm grade III placental calcification (diffuse echogenic lines or indentations extending from the chorionic plate to the basal layer of the placenta before 37 weeks' gestation) and high impedance of uterine arteries (pulsatility index $>1.2$ or the finding of a diastolic notch under uterine artery Doppler velocimetry), may be an implication of poor placental function and perinatal outcomes, and can serve as a marker of further intrapartum and postpartum events $[3,5,9,11,17-18]$. Using ultrasonography, we have previously shown that preterm grade III placental calcification is a major risk factor for progression of GH to PE [17], adverse maternal and neonatal outcomes [18, 19], and even stillbirth [20].

Some women with pregnancies complicated by GH-PE will have persistent postpartum chronic hypertension (CHTN) [8, 21]. Previous studies have revealed that older age, pre-existing chronic hypertension, smoking, pre-pregnancy obesity (elevated body mass index [BMI]), and co-morbidities such as thyroid disorders are common risk factors for postpartum CHTN [22, 23]. Obesity [10, 12, 16] and excessive weight gain [17] are risk factors for elevated $\mathrm{BP}$, resulting from chronic inflammation, oxidative stress, and placental endothelial cell activation $[10,12$, 16]. However, most of explored risk factors for CHTN are pre-pregnancy factors, based on studies with small sample sizes $(<2,000$ GH-PE women) $[22,23]$.
Thus, by analyzing a large population $(>20,000$ index pregnancies), the current study aimed to identify both pre-pregnancy and intra-pregnancy provoking factors for postpartum CHTN, in women with preceding GH-PE.

\section{Materials and methods}

This longitudinal cohort study was conducted in a tertiary teaching hospital with an average of $\geq 200$ deliveries per month. The study was approved by the Institutional Review Board of Taipei Tzu-Chi Hospital, Taiwan, followed the principle of the declaration of Helsinki, and met the guidelines of the responsible governmental agency.

During the period between 1, July, 2005 and 30, June, 2017, all eligible pregnancies of women in the obstetric clinics were enrolled for the study to decrease selection bias. Initial screening was performed to exclude pregnancies in women who had missing medical data, or did not deliver at our hospital. Patients were also excluded in case of inconsistent description for disease diagnosis and progression, and wrong or inappropriate coding of diagnoses and treatment. Because the current study focused on provoking factors for postpartum CHTN in GH-PE pregnancies, women who had pre-existing chronic hypertension were excluded from the study. The diagnosis of GH is made by $\geq 2$ measurements of hypertension (systolic $\mathrm{BP} \geq 140 \mathrm{mmHg}$ or diastolic $\mathrm{BP}$ $\geq 90 \mathrm{mmHg}$ ) noted at 20, 24, and 28 weeks gestation without proteinuria and not treated using anti-hypertensive medications, including hydralazine, methdopa, and nifedipine. According to Williams Obstetrics, PE is defined as hypertension during pregnancy (BP $\geq 140 / 90 \mathrm{mmHg}$ ) accompanied by proteinuria ( $\geq 300 \mathrm{mg} / 24 \mathrm{~h}$ or $\geq 1+$ on dipstick) [2]. The severity of GH-PE is classified by the occurrence of PE or not (GH alone).

Basic information including age, pre-pregnancy BMI, parity, smoking, general medical history and co-morbidities were retrieved at the first antepartum visit. Identification of multi-fetal pregnancy, diagnoses of pre-existing chronic hypertension, GH-PE and gestational diabetes mellitus (GDM) were made on subsequent antepartum visits. The diagnosis of persistent postpartum CHTN is confirmed by records of hypertension noted at both one month and one year after delivery. Excessive pregnant weight gain was defined as $\geq 10 \mathrm{kgw}$ of weight gain at 28 weeks when compared to 8 weeks, according to the recommendations for weight gain during pregnancy [2].

Using SPSS 19.0 (SPSS, Inc., Chicago, IL, USA), descriptive statistics, chi-square $\left(X^{2}\right)$ test, and Student t-test, were performed as appropriate to compare the 
characteristics and outcomes of pregnancies. Logistic regression analysis using stepwise selection method was performed to explore the provoking factors for postpartum CHTN by reporting odds ratios (ORs) and $95 \%$ confidence intervals (CIs) after adjusting for maternal age, pre-pregnancy BMI, parity, multi-fetal pregnancy, excessive pregnant weight gain, marital status, smoking, delivery type, severity of GH-PE and co-morbidities.

\section{Results}

A flow diagram for pregnancies classified according to blood pressure is shown in Figure 1. There were 25,068 pregnancies of women who underwent antepartum examinations at the obstetric clinics. After initial screening excluded women who had missing data or did not deliver at our hospital (n $=1,317), 23,751$ pregnancies were eligible for further analysis. A further 953 index pregnancies were excluded due to preexisting chronic hypertension, with 22,798 index pregnancies meeting the inclusion criteria. Of the 22,798 index pregnancies 2,132 were complicated by elevated BP (the GH-PE group) and the other 20,666 were presented with normal BP during antepartum examinations. Among the postpartum women in the GH-PE group, 428 (20.1\%) were complicated by chronic hypertension (the CHTN group), and 1,704 (79.9\%) had normal blood pressure (the NBP group).

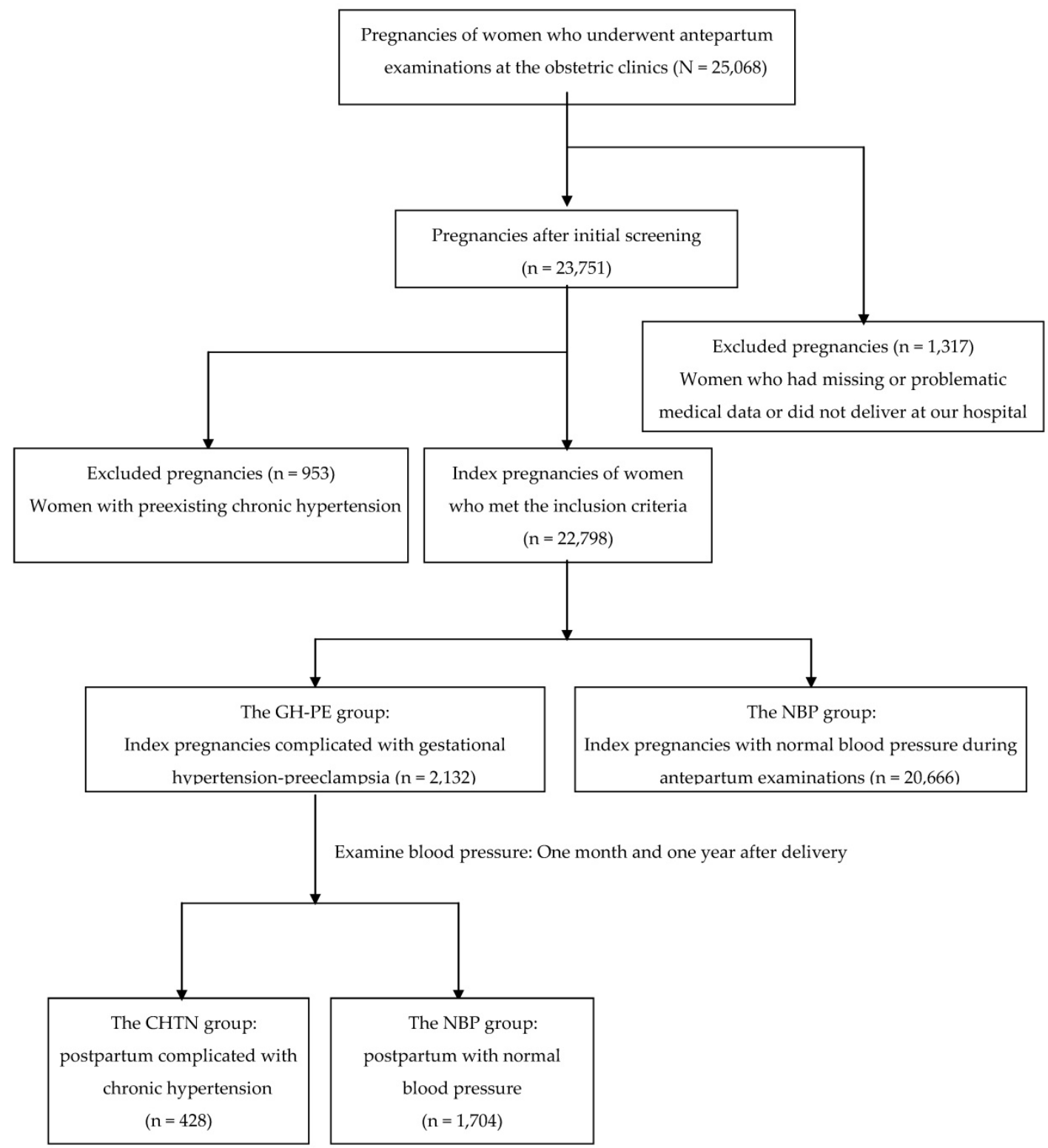

Figure 1. A flow diagram for pregnancies classified according to blood pressure. 
Table 1. Comparison of GH-PE pregnancies between persistent postpartum chronic hypertension (CHTN) and normal blood pressure (NBP) groups

\begin{tabular}{|c|c|c|c|c|c|c|c|}
\hline & \multicolumn{2}{|c|}{ CHTN group $(n=428)$} & \multicolumn{2}{|c|}{ NBP group $(n=1704)$} & \multicolumn{2}{|c|}{ Statistics } & \multirow[b]{2}{*}{$95 \% \mathrm{CI}$} \\
\hline & $\mathrm{N}$ & $\%$ & $\mathrm{~N}$ & $\%$ & $p$-value & $\mathrm{OR}^{\mathrm{a}}$ & \\
\hline & \multicolumn{2}{|c|}{$($ mean \pm SD) } & \multicolumn{2}{|c|}{$($ mean \pm SD) } & & & \\
\hline Age (years) & \multicolumn{2}{|c|}{$32.72 \pm 1.21$} & \multicolumn{2}{|c|}{$29.95 \pm 3.21$} & $<0.001^{* * *}$ & $1.80^{* * *}$ & $1.68-1.93$ \\
\hline Pre-pregnancy BMI $\left(\mathrm{kg} / \mathrm{m}^{2}\right)$ & \multicolumn{2}{|c|}{$23.51 \pm 1.09$} & \multicolumn{2}{|c|}{$22.48 \pm 1.24$} & $<0.001^{* * *}$ & $3.15^{\star \star *}$ & $2.75-3.60$ \\
\hline \multicolumn{5}{|l|}{ Parity } & 0.619 & & \\
\hline 0 & 27 & 63.8 & 1084 & 63.6 & & \multicolumn{2}{|l|}{ reference } \\
\hline 1 & 119 & 27.8 & 452 & 26.5 & & 1.03 & $0.80-1.32$ \\
\hline 2 or more & 36 & 8.4 & 168 & 9.9 & & 0.84 & $0.53-1.31$ \\
\hline \multicolumn{5}{|l|}{ Multi-fetal pregnancy } & 0.794 & & \\
\hline No & 408 & 95.3 & 1629 & 95.6 & & \multicolumn{2}{|l|}{ reference } \\
\hline Yes & 20 & 4.7 & 75 & 4.4 & & 1.07 & $0.65-1.77$ \\
\hline \multicolumn{5}{|l|}{ Marital status } & 0.934 & & \\
\hline No & 51 & 11.9 & 208 & 12.2 & & \multicolumn{2}{|l|}{ reference } \\
\hline Yes & 377 & 88.1 & 1496 & 87.8 & & 0.93 & $0.63-1.38$ \\
\hline \multicolumn{5}{|l|}{ Excessive pregnant weight gain ${ }^{\mathrm{b}}$} & $<0.001^{* * *}$ & & \\
\hline No & 85 & 19.9 & 1294 & 75.9 & & \multicolumn{2}{|l|}{ reference } \\
\hline Yes & 343 & 80.1 & 410 & 24.1 & & $14.50^{\star * *}$ & $11.02-19.08$ \\
\hline \multicolumn{5}{|l|}{ Smoking } & $0.012^{*}$ & & \\
\hline No & 342 & 79.9 & 1448 & 85.0 & & \multicolumn{2}{|l|}{ reference } \\
\hline Yes & 86 & 20.1 & 256 & 15.0 & & $1.79^{* * *}$ & $1.35-2.38$ \\
\hline \multicolumn{5}{|l|}{ Delivery type } & 0.511 & & \\
\hline Vaginal delivery & 256 & 59.8 & 987 & 57.9 & & \multicolumn{2}{|l|}{ reference } \\
\hline Cesarean delivery & 172 & 40.2 & 717 & 42.1 & & 0.94 & $0.75-1.17$ \\
\hline \multicolumn{5}{|l|}{ Severity of GH-PE } & $<0.001^{* * *}$ & & \\
\hline GH alone & 257 & 60.0 & 681 & 40.0 & & \multicolumn{2}{|l|}{ reference } \\
\hline PE & 171 & 40.0 & 1023 & 60.0 & & $2.46^{* * *}$ & $1.97-3.07$ \\
\hline \multicolumn{8}{|l|}{ Co-morbidities } \\
\hline Cardiovascular disease & 10 & 2.3 & 36 & 2.1 & 0.713 & 1.12 & $0.56-2.26$ \\
\hline Respiratory tract disease & 12 & 2.8 & 43 & 2.5 & 0.734 & 1.12 & $0.59-2.14$ \\
\hline Overt diabetes mellitus & 86 & 20.1 & 168 & 9.9 & $<0.001^{* * *}$ & $2.30^{\star * *}$ & $1.73-3.06$ \\
\hline Gestational diabetes mellitus & 257 & 60.0 & 330 & 19.4 & $<0.001^{* * *}$ & $6.25^{* * *}$ & $4.98-7.85$ \\
\hline Thyroid disease & 21 & 5.0 & 85 & 5.0 & 0.976 & 1.02 & $0.63-1.66$ \\
\hline Renal disease & 22 & 5.3 & 84 & 4.9 & 0.802 & 1.08 & $0.67-1.75$ \\
\hline
\end{tabular}

Data are expressed as the number (\%) or mean \pm standard deviation (SD), as appropriate

${ }^{*} p<0.05,{ }^{* *} p<0.01,{ }^{* * *} p<0.01$, by chi-square test, Student's t-test, or logistic regression analysis, as appropriate

a Logistic regression analysis of adjusted odds ratio (OR) and 95\% confidence interval (CI) for the occurrence of CHTN, compared with the reference (NBP) group

b Weight gain $\geq 10 \mathrm{kgw}$ during pregnancy, measured at 28 weeks' gestation

The characteristics and co-morbidities of the GH-PE pregnancies between the postpartum CHTN and NBP groups are compared in Table 1 . There were significant differences in maternal age, pre-pregnancy $\mathrm{BMI}$, the ratios of excessive pregnant weight gain, severity of GH-PE, overt DM and GDM (all $p<0.001$ ), and smoking $(p<0.05)$ between the two groups. Logistic regression analysis was used to explore provoking factors for the occurrence of postpartum CHTN in GH-PE women. After adjustment, older maternal age $(\mathrm{OR}=1.80 ; 95 \% \mathrm{CI}=1.68-1.93)$, elevated pre-pregnancy BMI (OR=3.15; 95\% $\mathrm{CI}=2.75-3.60)$, excessive pregnant weight gain (OR=14.50; 95\% $\mathrm{CI}=11.02-19.08)$, cigarette smoking ( $\mathrm{OR}=1.79 ; 95 \%$ $\mathrm{CI}=1.35-2.38)$, severity of $\mathrm{GH}-\mathrm{PE} \quad(\mathrm{OR}=2.46 ; 95 \%$ $\mathrm{CI}=1.97-3.07)$, overt $\mathrm{DM}(\mathrm{OR}=2.30 ; 95 \% \mathrm{CI}=1.73-3.06)$ and GDM $(\mathrm{OR}=6.25 ; 95 \% \quad \mathrm{CI}=4.98-7.85)$ were associated with an increased risk of postpartum CHTN. Remarkably, excessive pregnant weight gain and GDM were two major provoking factors for postpartum CHTN.

\section{Discussion}

By excluding women with pre-existing chronic hypertension, this current study focused mainly on the effect of pregnancy on subsequent postpartum CHTN. Compared to the incidence $(821 / 20,666=$ $3.97 \%$ ) of postpartum CHTN in normotensive pregnancies, women which developed hypertension during pregnancy (GH-PE) had a near five-fold incidence $(20.1 \%)$ of postpartum CHTN. Moreover, our results revealed that intra-pregnancy factors (excessive pregnant weight gain, GDM and severity of GH-PE) had greater effects on postpartum CHTN, compared with pre-pregnancy factors (pre-pregnancy BMI, smoking, pre-existing overt DM and other co-morbidities). Thus women with excessive pregnant weight gain or GDM should be screened for hypertension after delivery, in order to allow for prompt management.

The result of the current study revealed that both excessive pregnant weight gain and pre- pregnancy obesity are risk factors for postpartum CHTN. The mechanisms of obesity-associated HTN are complex. 
Obesity and excessive deposition of adipose tissues may alter endothelial function and enhance BP via chronic inflammation, oxidative stress, sodium retention, insulin resistance and placental endothelial cell activation [10, 12-17, 24]. Adipose tissue of obese patients becomes resistant to insulin and is the site of altered secretion of molecules such as adiponectin, leptin, resistin, tumor necrosis factor (TNFa) and IL-6, which induce obesity-associated cardiovascular disease [13, 14]. During the early phases of obesity, increased renal tubular reabsorption leads to primary sodium retention. Extracellular-fluid volume is expanded and the kidney-fluid apparatus is resetted to a hypertensive level, consistent with a volume overload-hypertension model [14, 15]. Plasma renin activity, angiotensinogen, angiotensin II (AngII) and aldosterone values display significant increase during obesity and DM [14, 15]. Obesity-associated microvascular dysfunction and resultant HTN is thought to arise via two signaling pathways. In the endothelial cells, insulin-mediated PI3-kinase (PI3K)/NO activation leads to vasodilation. In contrast, endothelin 1 (ET-1) production activates the MAPK pathway, leading to vasoconstriction [14, 25]. The mediators AngII, TNFa, and free fatty acids (FFA), which are released from the adipose tissues of obese patients, can inhibit the PI3-kinase (PI3K) pathway and stimulate the MAPK pathway [25]. In pregnant women with excessive weight gain, increased sodium retention, and metabolic disturbances such as enhanced oxidative stress and insulin resistance, may underlie their predisposition to develop postpartum CHTN [10, 12-14, 16, 25].

GDM was also found to be a significant predictor for postpartum CHTN in the current study. Elevated BP in GDM patients appears to be closely related to increased circulatory fluid volume and increased peripheral vascular resistance $[15,26]$. Specifically, pregnant patients with diabetes mellitus (including overt DM and GDM) experience increased peripheral artery resistance and increased body fluid volume, secondary to hyperinsulinemia and hyperglycemia. Both of these mechanisms elevate systemic BP $[15,26]$.

In comparison to $\mathrm{GH}$ alone, the occurrence of $\mathrm{PE}$ is associated with more profuse systemic impairment, including vascular endothelial injury to the cardiovascular system [1-3, 27]. Our result is similar to another research reporting increased risk of subsequent hypertension among pregnant women with more severe PE [27]. In addition, cigarette smoking $(\mathrm{OR}=1.79)$ is a provoking factor for postpartum CHTN, and the finding is consistent with our previous report which demonstrated that cigarette smoking slightly increased the risk of $\mathrm{GH}$ and PE (OR=1.42 1.68) [17]. It is well known that cigarette smoking can result in generalized endothelial dysfunction. Hence, cigarette smoking cessation is helpful to prevent both GH-PE and subsequent CHTN [17].

Compared with previous reports that focused mainly on pre-pregnancy factors, the current report is the first study to investigate both pre-pregnancy and intra-pregnancy factors, using a large cohort $(>20,000$ index pregnancies). Importantly, our results suggest modifiable risk factors such as excessive pregnant weight gain and GDM are associated with increased risk for postpartum CHTN. The limitation of our study arises from some characteristics such as race and socio-economic status, which were not considered and could affect the results. Currently, there are still many potential factors for predisposing to persistent postpartum $\mathrm{CHTN}$, which remain unexplored and warrant further investigation.

\section{Conclusion}

Excessive pregnant weight gain and GDM are major intra-pregnancy risk factors for postpartum CHTN in women with preceding GH-PE. Women with excessive pregnant weight gain or GDM should be screened for hypertension after delivery. Future studies should investigate interventions such as a healthy diet, appropriate physical exercise and avoidance of excessive pregnant weight gain as a means to reduce the frequency of CHTN following pregnancy.

\section{Acknowledgements}

\section{Funding}

This research is funded by a grant from Taipei Tzu-Chi Hospital, The Buddhist Tzu-Chi Medical Foundation, Taipei, Taiwan (TCRD-TPE-109-10).

\section{Author Contributions}

K.H.C. conceived this study and collected the data. K.H.C. and L.R.C. participated in the analysis and interpretation of the results. K.H.C. and L.R.C. contributed to writing the article. Both authors reviewed and approved the final version of the report.

\section{Competing Interests}

The authors have declared that no competing interest exists.

\section{References}

1. Sibai BM. Diagnosis and management of gestational hypertension and preeclampsia. Obstet Gynecol. 2003; 102: 181-92.

2. Cunningham FG, Leveno KJ, Bloom SL, et al. Prenatal care, Pregnancy hypertension and Obesity. In Cunningham FG (ed), Williams Obstetrics. New York, McGraw Hill, 2010, pp.176-7, 706-14, 964-5.

3. Mol BW, Roberts CT, Thangaratinam S, et al. Pre-eclampsia. Lancet. 2016; 387: 999-1011. 
4. World Health Organization. Make Every Mother and Child Count. World Health Report. Geneva Switzerland, 2005.

5. Visentin S, Londero AP, Bellamio B, et al. Fetal endothelial remodeling in late-onset gestational hypertension. Am J Hypertens. 2016; 29: 273-9.

6. Visentin S, Grisan E, Zanardo V, et al. Developmental programming of cardiovascular risk in intrauterine growth-restricted twin fetuses according to aortic intima thickness. J Ultrasound Med. 2013; 32: 279-84.

7. Veerbeek JH, Hermes W, Breimer AY, et al. Cardiovascular disease risk factors after early-onset preeclampsia, late-onset preeclampsia, and pregnancy-induced hypertension. Hypertension. 2015; 65: 600-6.

8. Melchiorre K, Sutherland GR, Liberati M, et al. Preeclampsia is associated with persistent postpartum cardiovascular impairment. Hypertension. 2011; 58: 709-15.

9. Farag K, Hassan I, Ledger WL. Prediction of preeclampsia: can it be achieved? Obstet Gynecol Surv. 2004; 59: 464-82.

10. Spradley FT, Palei AC, Granger JP. Immune mechanisms linking obesity and preeclampsia. Biomolecules. 2015; 5: 3142-76.

11. Prefumo F, Sebire NJ, Thilaganathan B. Decreased endovascular trophoblast invasion in first trimester pregnancies with high-resistance uterine artery Doppler indices. Hum Reprod. 2004; 19: 206-9.

12. Wolf M, Kettyle E, Sandler L, et al. Obesity and preeclampsia: the potential role of inflammation. Obstet Gynecol. 2001; 98(5 Pt 1): 757-62.

13. DeMarco VG, Aroor AR, Sowers JR. The pathophysiology of hypertension in patients with obesity. Nat Rev Endocrinol. 2014; 10: 364-76

14. Kotsis V, Stabouli S, Papakatsika S, et al. Mechanisms of obesity-induced hypertension. Hypertens Res. 2010; 33: 386-93.

15. Cheung BM, Li C. Diabetes and hypertension: is there a common metabolic pathway? Curr Atheroscler Rep. 2012; 14: 160-6.

16. Zavalza-Gomez AB. Obesity and oxidative stress: a direct link to preeclampsia? Arch Gynecol Obstet. 2011; 283: 415-22.

17. Chen KH, Seow KM, Chen LR. Progression of gestational hypertension to pre-eclampsia: A cohort study of 20,103 pregnancies. Pregnancy Hypertens. 2017; 10: $230-7$.

18. Chen $\mathrm{KH}$, Chen LR, Lee YH. Exploring the relationship between preterm placental calcification and adverse maternal and fetal outcome. Ultrasound Obstet Gynecol. 2011; 37: 328-34.

19. Chen $\mathrm{KH}$, Chen LR, Lee $\mathrm{YH}$. The role of preterm placental calcification in high-risk pregnancy as a predictor of poor uteroplacental blood flow and adverse pregnancy outcome. Ultrasound Med Biol. 2012; 38: 1011-8.

20. Chen KH, Seow KM, Chen LR. The role of preterm placental calcification on assessing the risks of stillbirths. Placenta. 2015; 36: 1039-44.

21. Lykke JA, Langhoff-Roos J, Sibai BM, et al. Hypertensive pregnancy disorders and subsequent cardiovascular morbidity and type 2 diabetes mellitus in the mother. Hypertension. 2009; 53: 944-51.

22. Hwang JW, Park SJ, Oh SY, et al. The risk factors that predict chronic hypertension after delivery in women with a history of hypertensive disorders of pregnancy. Medicine. 2015; 94: e1747.

23. Takaoka S, Ishii $K$, Taguchi $T$, et al. Clinical features and antenatal risk factors for postpartum-onset hypertensive disorders. Hypertens Pregnancy. 2016; 35: 22-31.

24. Aliyu MH, Luke S, Kristensen S, et al. Joint effect of obesity and teenage pregnancy on the risk of preeclampsia: a population-based study. J Adolesc Health. 2010; 46: 77-82

25. Jonk AM, Houben AJHM, de Jongh RT, et al. Microvascular dysfunction in obesity: a potential mechanism in the pathogenesis of obesity-associated insulin resistance and hypertension. Physiology. 2007; 22: 252-60.

26. Ohishi M. Hypertension with diabetes mellitus: physiology and pathology. Hypertens Res. 2018; 41: 389-93.

27. Best LG, Lunday L, Webster E, et al. Pre-eclampsia and risk of subsequent hypertension: in an American Indian population. Hypertens Pregnancy. 2017; 36: 131-7. 